August 2019. Title, abstract, and full text screening were conducted following the Preferred Reporting Items for Systematic Reviews and Meta-Analyses. The target population was neonates born during humanitarian emergencies. Studies from high-income countries and prior to 1991 were excluded. The STROBE checklist was used to assess for risk of bias.

Results A total of 35 articles were included in the analysis; these were mainly qualitative, field-based studies. The primary needs identified were referrals from homes to health facilities before and during labour, and inter-facility referrals after labour to more specialised services. Some of the main barriers included a lack of roads and infrastructure for transport, a deficiency of specialised equipment for complications, staff shortages - especially among more specialised services, and a lack of knowledge among healthcare workers for identifying neonatal referral issues. Effective mechanisms for addressing these needs and gaps included establishing pre- or post-natal home visits by Community Health Workers or Traditional Birth Attendants; standardised, inter-facility referral checklists and procedures for healthcare staff; and staff training programmes for identification of neonatal complications. The severity and issues varied significantly between different countries and emergencies.

Conclusion This review benefited from a strong consensus among selected studies but was limited in the quality of data and types of data that were reported. Based on the above findings, the following recommendations were compiled:

- Recruit community healthcare workers to raise awareness and reduce pressure on health facilities.

- Establish contextualised programmes that address local needs and draw on local resources.

- Continue to train healthcare workers to provide timely, appropriate and quality care during humanitarian emergencies.

\section{G465(P) TACKLING LOCAL OBSTACLES TO EFFECTIVE KANGAROO MOTHER CARE IN A DISTRICT HOSPITAL IN RURAL RWANDA: AN OBSERVATIONAL STUDY}

${ }^{1} \mathrm{~T}$ Slater, ${ }^{2}$ E Smeaton-Russell, ${ }^{3} \mathrm{~T}$ Lissauer. ${ }^{1}$ Specialist Registrar, South Thames Deanery, London, UK; ${ }^{2}$ Paediatric Nurse, Great Ormond Street Hospital, London, UK; ${ }^{3}$ Hon. Consultant Neonatologist, Imperial College Healthcare Trust, London, UK

\subsection{6/archdischild-2020-rcpch.399}

Aims To identify obstacles to effective Kangaroo Mother Care (KMC) at a district hospital in rural Rwanda.

Background KMC can improve thermoregulation and weight gain, whilst reducing infections and ultimately mortality. It can provide a safe alternative to incubator care for stable babies. Whilst KMC is often an established facility in Rwanda hospitals, local barriers may exist to limit the desired practice and outcomes in infants.

Methods Over a 2-week period at the start of a 6-month placement, detailed observations of KMC practice 3 times a day were made.

Results were collated and discussed with medical and nursing staff working in the neonatal unit. A programme to overcome key, local barriers to effective KMC was then devised.
Results Key barriers and initiatives to overcome them were:

- Poor maternal nutrition - extreme local poverty and geographical isolation of the hospital on a mountain led to limited social support for mothers. Prolonged absence (and even absconding) of mothers was necessary to source food. Provision of a water filter and creation of a local egg cooperative helped to improve nutrition and minimise time needed away from their babies. With support for the cooperative from the adjacent church, free eggs are now provided daily to long-term mothers in the unit.

- Lack of space - a new, larger room for mothers undertaking KMC was sourced with our supervision, as part of a planned hospital re-organisation. This provided more space and independent washing facilities for mothers, creating a more pleasant environment.

- Lack of facilities -sourcing locally produced comfortable chairs and appropriate storage for belongings encouraged mothers to spend more effective time with their babies in KMC.

- Limited nursing staff - other members of the hospital staff, including social workers were mobilised to run education sessions on neonatal 'red flags' (i.e. fever, jaundice, lethargy, poor feeding). Basic educational materials and posters were provided to facilitate this.

Conclusion Detailed observation followed by staff engagement allowed many of the obstacles to effective KMC to be overcome with low cost interventions. Nevertheless, innovative and local initiatives are critical to achieving major improvements.

\section{G466(P) TRAINING ON THE RECOGNITION OF THE SICK NEONATE}

${ }^{1} \mathrm{E}$ MacRae, ${ }^{2} \mathrm{~T}$ Kyaw, ${ }^{3} \mathrm{SM}$ Thu, ${ }^{4} \mathrm{~L}$ Davidson. ${ }^{1}$ Paediatrics, RCPCH, London, UK; ${ }^{2}$ Paediatrics, Myanmar Paediatric Society, Hinthada, Myanmar; ${ }^{3}$ Paediatrics, Myanmar Paediatric Society, Mawgyun, Myanmar; ${ }^{4}$ Paediatric Nursing, Royal College of Nursing, London, UK

\subsection{6/archdischild-2020-rcpch.400}

Background As part of the partnership with Myanmar paediatric society and the Royal College of paediatrics, work has been undertaken to improve child and neonatal mortality. Our aim was to increase vital sign monitoring; the recognition of the sick or deteriorating neonate and timely escalation of care. To achieve this objective, a training programme was devised for nursing staff to increase awareness of neonatal danger signs and when to inform the doctors of the patient's condition.

Objective To determine if the training programme for nursing staff was effective in improving their awareness of neonatal danger signs and when to inform the doctors of the patient's condition. This was determined by comparing their mean test scores before and after the programme for any statistically significant improvement.

Method The training was carried out amongst 17 nurses (paediatric, neonatal and nursing students) in two hospitals in the Ayeyarwady region (Hospital A and Hospital B). A poster was created detailing how to monitor the neonates, basic emergency management of sick neonates for the nursing staff and when to escalate concerns to a paediatric doctor. 